\title{
OPEN Exercise training mitigates ER stress and UCP2 deficiency-associated coronary vascular dysfunction in atherosclerosis
}

\author{
Junyoung Hong ${ }^{1}$, Eunkyung Park ${ }^{1}$, Jonghae Lee ${ }^{1}$, Yang Lee ${ }^{2}$, Bridgette V. Rooney ${ }^{1,3}$ \& \\ Yoonjung Park ${ }^{1 \bowtie}$
}

Endoplasmic reticulum (ER) stress and uncoupling protein-2 (UCP2) activation are opposing modulators of endothelial dysfunction in atherosclerosis. Exercise reduces atherosclerosis plaques and enhances endothelial function. Our aim was to understand how exercise affects ER stress and UCP2 activation, and how that relates to endothelial dysfunction in an atherosclerotic murine model. Wild type (C57BL/6, WT) and apolipoprotein-E-knockout (ApoE ${ }^{\text {tm1Unc }}$, ApoE KO) mice underwent treadmill exercise training (EX) or remained sedentary for 12 weeks. Acetylcholine (ACh)-induced endothelium-dependent vasodilation was determined in the presence of an eNOS inhibitor (L-NAME), UCP2 inhibitor (genipin), and ER stress inducer (tunicamycin). UCP2, ER stress markers and NLRP3 inflammasome signaling were quantified by western blotting. $\mathrm{p} 67^{\text {phox }}$ and superoxide were visualized using immunofluorescence and DHE staining. Nitric oxide (NO) was measured by nitrate/nitrite assay. ACh-induced vasodilation was attenuated in coronary arterioles of ApOE KO mice but improved in ApoE KO-EX mice. Treatment of coronary arterioles with L-NAME, tunicamycin, and genipin significantly attenuated $\mathrm{ACh}$-induced vasodilation in all mice except for ApoE KO mice. Exercise reduced expression of ER stress proteins, TXNIP/NLRP3 inflammasome signaling cascades, and Bax expression in the heart of ApOE KO-EX mice. Further, exercise diminished superoxide production and NADPH oxidase $\mathrm{p} 67^{\text {phox }}$ expression in coronary arterioles while simultaneously increasing UCP2 expression and nitric oxide (NO) production in the heart of ApOE KO-EX mice. Routine exercise alleviates endothelial dysfunction in atherosclerotic coronary arterioles in an eNOS, UCP2, and ER stress signaling specific manner, and resulting in reduced TXNIP/NLRP3 inflammasome activity and oxidative stress.

Atherosclerosis is a major risk factor for cardiovascular disease and is one of the leading causes of death in the United States ${ }^{1}$. It is a chronic inflammatory disease of both small and large arteries and is characterized by largescale lipid plaques in vessel walls ${ }^{2}$. These plaques are responsible for both mechanical and physiological reductions in endothelial function. Endothelial dysfunction is considered a hallmark indicator of the development and progression of atherosclerosis, and is associated with reductions in NO bioavailability along with increases in inflammation, reactive oxygen species (ROS) and apoptosis around the atherosclerotic lesion ${ }^{3}$.

Endoplasmic reticulum (ER) stress precipitates the initiation and progression of atherosclerosis and modulates endothelial dysfunction by influencing eNOS signaling pathway regulation ${ }^{4}$. Impaired ER homeostasis, due to many factors including hypercholesteremia and hyperlipidemia, interrupts normal protein folding in the ER lumen, resulting in aberrant protein conformations that trigger the unfolded protein response (UPR). The UPR is regulated by three crucial ER membrane proteins: PKR-like endoplasmic reticulum kinase (PERK), inositolrequiring enzyme 1 (IRE1), and activating transcription factor 6 (ATF6). Persistent UPR activity induces ER

\footnotetext{
${ }^{1}$ Department of Health and Human Performance, Laboratory of Integrated Physiology, University of Houston, 3875 Holman St, Houston, TX 77204-6015, USA. ²Department of Medical Physiology, College of Medicine, Texas A\&M University, College Station, TX 77807, USA. ${ }^{3}$ Geocontrol Systems Inc, NASA Johnson Space Center, Houston, TX 77058, USA. ${ }^{\square}$ email: ypark10@uh.edu
} 


\begin{tabular}{|l|l|l|l|l|}
\hline \multirow{2}{*}{} & \multicolumn{2}{|l|}{ Sedentary } & \multicolumn{2}{l|}{ Exercise } \\
\cline { 2 - 6 } & WT & ApoE KO & WT-EX & ApoE KO-EX \\
\hline $\mathrm{N}$ & 10 & 9 & 6 & 7 \\
\hline Coronary arterioles initial lumen diameter, $\mu \mathrm{m}$ & $81.88 \pm 6.04$ & $75.70 \pm 11.33$ & $95.53 \pm 6.70$ & $91.78 \pm 8.24$ \\
\hline Coronary arterioles maximal lumen diameter, $\mu \mathrm{m}$ & $125.89 \pm 11.31$ & $116.99 \pm 8.87$ & $126.14 \pm 7.68$ & $119.79 \pm 7.79$ \\
\hline
\end{tabular}

Table 1. Vessel characteristics. The initial lumen diameter and maximal lumen diameter of coronary arterioles were measured at 24-25 weeks of age.

stress, which may trigger increases in oxidative stress, IRE1-mediated inflammation, and CCAAT-enhancerbinding protein homologous protein (CHOP)-mediated apoptosis signaling ${ }^{4}$.

Excessive reactive oxygen species (ROS) production that overwhelms the antioxidant defense systems is one of the major contributing factors for endothelial dysfunction, and may lead to damage of the vascular endothelium ${ }^{5}$ and results in significant reductions of NO bioavailability in atherosclerosis ${ }^{6}$. Uncoupling protein-2 (UCP2) is a mitochondrial inner membrane protein that serves as an important negative regulator of mitochondrial-derived ROS production ${ }^{6}$. Consequently, it has emerged as an important antioxidant that prevents the development of atherosclerosis ${ }^{7}$, as well as ameliorates endothelial dysfunction by increasing NO bioavailability in metabolic disorders ${ }^{8}$.

Recently, it has been reported that thioredoxin-interacting protein (TXNIP) is a key factor linking ER stress to oxidative stress and inflammation ${ }^{9}$. It is also associated with endothelial dysfunction and the development of atherosclerosis ${ }^{10}$. The nucleotide-binding oligomerization domain (NOD)-like receptor pyrin domain-containing 3 (NLRP3) inflammasome consists of a multimeric protein complex: NLRP3, apoptosis-associated speck-like protein containing (ASC), and caspase-1. The NLRP3 inflammasome is a pivotal regulator of initial immune responses ${ }^{11}$ and is activated by TXNIP in response to ER stress ${ }^{12}$. Activation of the inflammasome promotes the maturation and secretion of both IL-1 $\beta$ and IL-18 in a caspase- 1 dependent manner ${ }^{13}$. Each of these cytokines, as well as activation of the NLRP3 inflammasome itself, has been implicated in endothelial dysfunction ${ }^{13,14}$ and atherosclerosis pathogenesis ${ }^{15}$. However, little is known about the mechanistic link between ER stress, TXNIP/ NLRP3 inflammasome activation, and UCP2 activity on coronary vascular dysfunction in atherosclerosis.

The cardiovascular benefits of exercise have been well documented to include improvements in endothelial function, and reductions in both inflammation and oxidative stress in atherosclerosis ${ }^{16}$. A handful of studies have attributed the benefit of routine exercise to the reduction in ER stress by highlighting that ER stress is associated with endothelial dysfunction in diabetes ${ }^{17}$ and atherosclerosis ${ }^{18}$. Additionally, previous studies from our lab have determined that vascular function is improved by routine exercise due to UCP2-mediation in atherosclerosis ${ }^{18}$ and decreases of NLRP3 inflammasome activity in obesity ${ }^{19}$. However, no study to date has sought to determine the combined interaction of ER stress, UCP2 expression, and NLRP3 activity in response to exercise in a murine atherosclerotic model.

Therefore, the main purpose of this study was to determine the possible underlying mechanisms responsible for the effect of exercise on ER stress and UCP2 deficiency-associated coronary vascular dysfunction in atherosclerosis. To answer these questions, we first evaluated whether ACh-induced vasodilation is impaired in coronary arterioles in atherosclerosis, and then we determined whether exercise training could improve coronary function by modulating the ER stress and UCP2 pathways, including the TXNIP/NLRP3 inflammasome cascade and oxidative stress response in atherosclerosis. We hypothesized that exercise training would mitigate endothelial dysfunction in atherosclerotic coronary arterioles through elevation of NO bioavailability. Also, we hypothesized that exercise training would alleviate ER stress-associated endothelial dysfunction in atherosclerotic coronary arterioles by reducing ER stress signaling and its downstream cascade including TXNIP/NLRP3 inflammasome. We further hypothesized that exercise training would mitigate UCP2 deficiency-associated vascular dysfunction through the restoration of UCP2 expression and reduction of oxidative stress in coronary arterioles of atherosclerosis.

\section{Results}

Animal and vascular characteristics. The animal characteristics have been presented in our previous study ${ }^{18}$. Neither initial nor maximal intraluminal diameters of coronary arterioles were significantly different in WT and atherosclerotic ApoE KO, and exercise training did not affect the diameters in either group (Table 1).

Exercise training ameliorates endothelial dysfunction via an increase in NO concentration in coronary arterioles of ApoE KO mice. ACh-induced endothelium-dependent vasodilation was significantly attenuated in coronary arterioles in ApoE KO mice compared with WT mice $(p<0.05$; Fig. 1A). However, exercise training significantly improved ACh-induced vasodilation in coronary arterioles of ApoE KO-EX mice compared to ApoE KO mice $(p<0.05$; Fig. $1 \mathrm{~A})$. Figure $1 \mathrm{~B}$ shows that the SNP-induced vasodilatory response was identical in the coronary arterioles of all mouse groups $(p>0.05)$.

Treatment of isolated coronary arterioles with L-NAME significantly attenuated vasodilation in response to ACh in the WT and ApoE KO-EX mice $(p<0.05)$, but not in ApoE KO mice (Fig. 1C,D). Furthermore, the absolute concentration of total nitrate/nitrite levels was significantly reduced in the heart of ApoE KO compared with WT $(p<0.05)$, but its level was significantly elevated in response to exercise training in the heart of ApoE KO-EX mice compared with ApoE KO mice (Fig. 1E). 

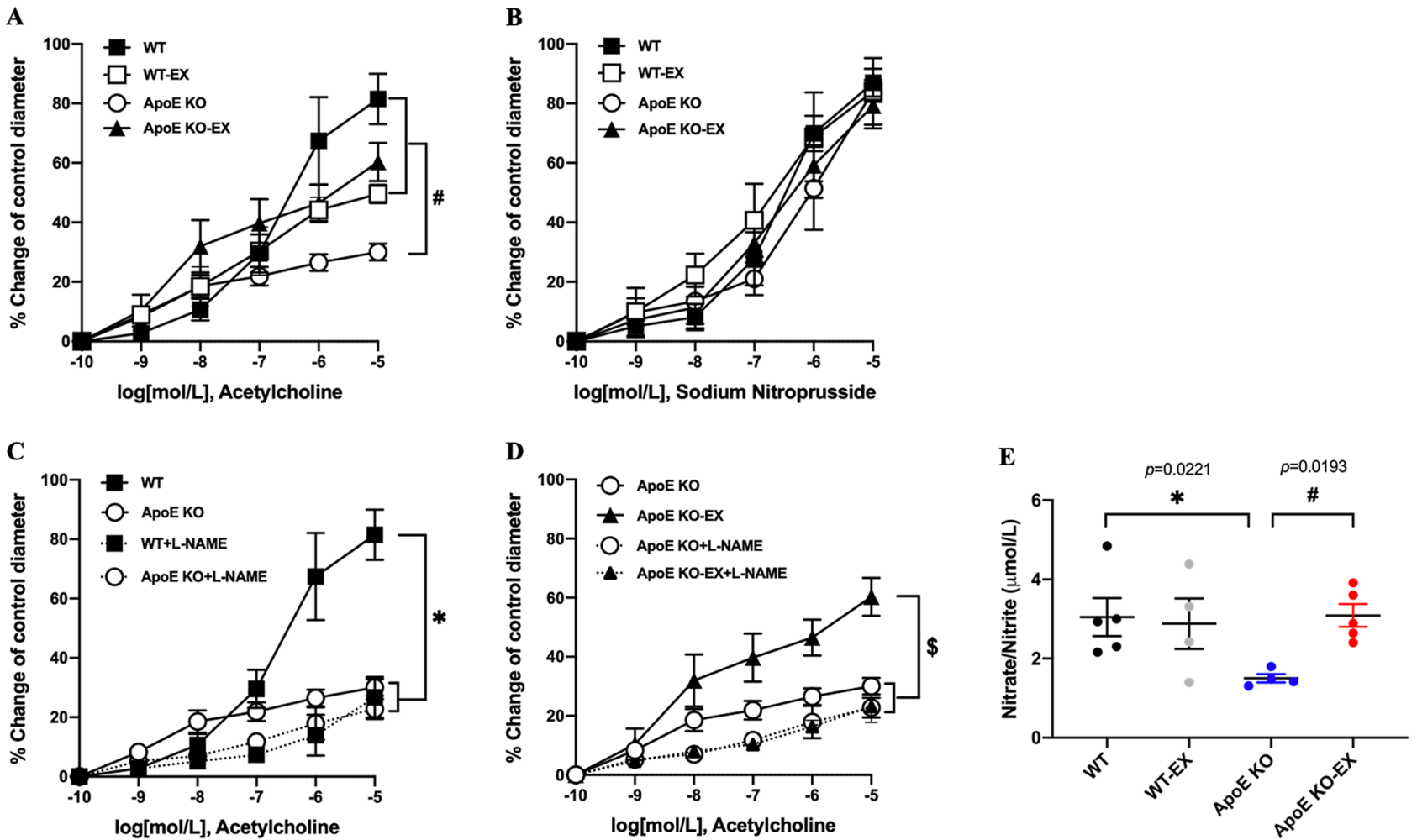

Figure 1. Effect of exercise training on endothelial dysfunction and NO production in ApoE KO mice. (A): Isolated coronary arterioles from WT $(\mathrm{n}=4)$, WT-EX $(\mathrm{n}=4)$, ApoE KO $(\mathrm{n}=5)$, and ApoE KO-EX $(\mathrm{n}=4)$ were measured the response of ACh in a dose-dependent manner. (B): Endothelium-independent vasodilation was measured in isolated coronary arterioles from WT $+\mathrm{SNP}(\mathrm{n}=5), \mathrm{WT}-\mathrm{EX}+\mathrm{SNP}(\mathrm{n}=4), \mathrm{ApoE} \mathrm{KO}+\mathrm{SNP}$ $(n=5)$, ApoE KO-EX+SNP $(n=4)$. $(\mathbf{C}, D)$ : ACh-induced vasodilation in coronary arterioles was measured in the presence of L-NAME, the eNOS inhibitor, from WT + L-NAME $(n=4)$, WT-EX+L-NAME $(n=4)$, ApoE $\mathrm{KO}+\mathrm{L}-\mathrm{NAME}(\mathrm{n}=5)$, and ApoE KO-EX + L-NAME $(\mathrm{n}=4) .(\mathrm{E})$ : Total nitrate/nitrite concentration was analyzed in mice heart from each group ( $\mathrm{n}=4-5$ /groups). Values are means \pm SEM. ${ }^{*} p<0.05$ versus $\mathrm{WT},{ }^{*} p<0.05$ versus ApoE KO, ${ }^{s} p<0.05$ versus ApoE KO-EX.

Exercise training mitigates ER stress-associated coronary vascular dysfunction in ApoE KO mice. Figure $2 \mathrm{~A}, \mathrm{~B}$ show that incubation with ER stress inducer (Tunicamycin) significantly impaired AChinduced vasodilation in coronary arterioles in both WT and ApoE KO-EX mice $(p<0.05)$, whereas no further deterioration was shown in coronary arterioles in ApoE KO mice. Likewise, the expression of ER stress markers including GRP78, p/t-IRE1, p/t-eIF2 $\alpha$, and CHOP were significantly increased in the heart of ApoE KO mice compared with WT mice $(p<0.05$; Fig. $2 \mathrm{C}-\mathrm{H})$, but up-regulated expression of these ER stress markers were significantly reduced by exercise training in the heart of ApoE KO-EX mice $(p<0.05 ;$ Fig. $2 \mathrm{C}-\mathrm{H})$. Finally, the elevated expression of the pro-apoptotic marker Bax was also significantly diminished in the heart of ApoE KO mice in response to exercise training $(p<0.05$; Fig. 2I).

Exercise training reduces the expression of TXNIP/NLRP3 inflammasome in the heart of ApoE KO mice. The expression of TXNIP, NLRP3, pro-caspase-1, caspase-1 p20, and IL-1 $\beta$ were significantly elevated in the heart of ApoE KO mice compared with WT mice, and exercise training significantly reduced the expression of TXNIP, caspase-1 p20, and IL- $1 \beta$ expression in the heart of ApoE KO-EX mice $(p<0.05$; Fig. 3A-E).

Exercise training mitigates UCP2 deficiency-mediated endothelial dysfunction in coronary arterioles and oxidative stress in heart of ApoE KO mice. Figure 4A,B show that treatment with UCP2 inhibitor (genipin) significantly attenuated ACh-induced vasodilation in coronary arterioles in both WT and ApoE KO-EX, but no further impairment was shown in coronary arterioles in ApoE KO mice. Thus, protein expression of UCP2 was significantly reduced in the heart of ApoE KO mice compared to WT mice, but its expression was increased by exercise training $(p<0.05$; Fig. 4 C).

The $\mathrm{O}_{2}^{-}$production was increased in coronary arterioles of ApoE KO heart compared with WT, but was diminished by exercise training shown by DHE staining $(p<0.05$; Fig. $4 \mathrm{D}, \mathrm{E})$. Immunofluorescence images demonstrated that NADPH oxidase subunit p67 $7^{\text {phox }}$ was abundantly expressed in endothelial cells of coronary arterioles in ApoE KO mice, and its expression appeared to be reduced by exercise training in ApoE KO-EX (Fig. 4F). 
A

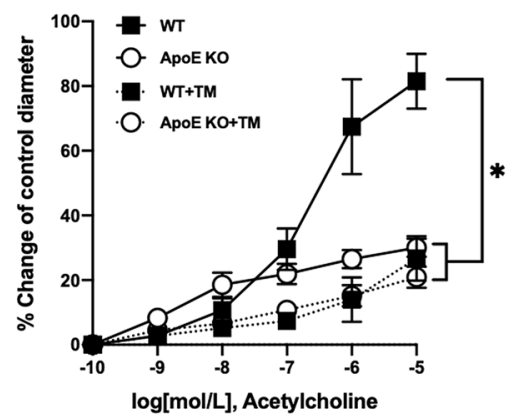

C

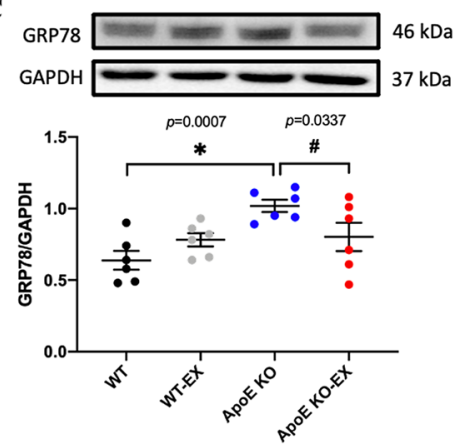

B

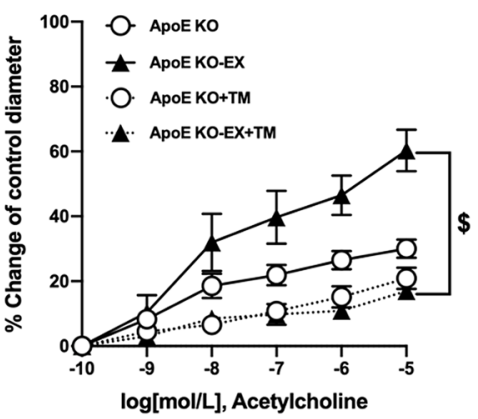

D

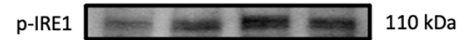

GAPDH $=-2 \mathrm{kDa}$

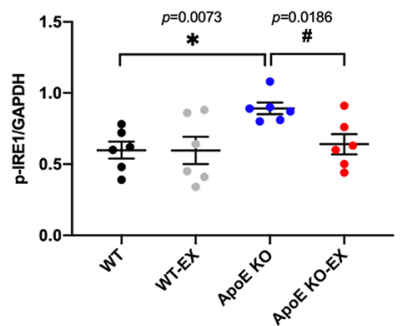

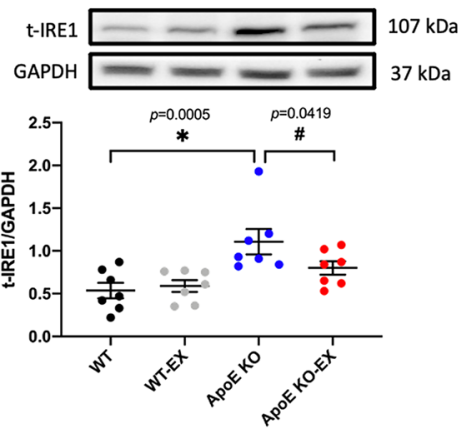

H

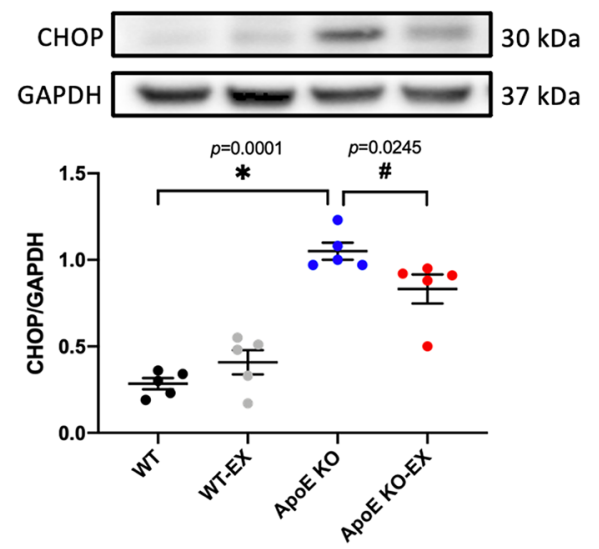

F
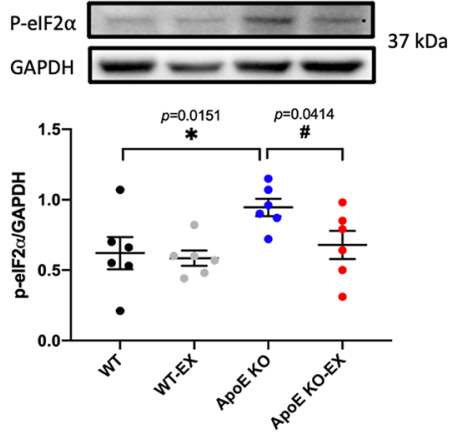

G

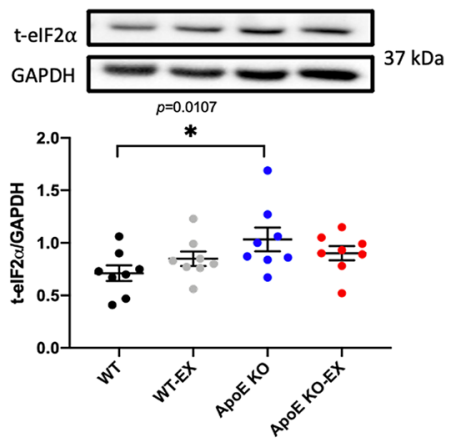

I

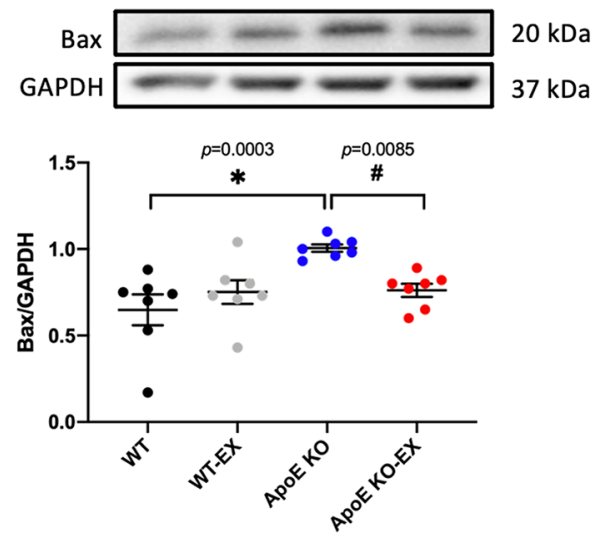

Figure 2. Effect of exercise training on ER stress-associated endothelial dysfunction in coronary arterioles and the expression of ER stress and apoptosis in the heart of ApoE KO mice. (A-B): ACh-induced vasodilation was measured in the presence of ER stress inducer (tunicamycin) from the isolated coronary arterioles in WT $+\mathrm{TM}$ $(\mathrm{n}=4)$, WT-EX+TM $(\mathrm{n}=4), \operatorname{ApoE~KO}+\mathrm{TM}(\mathrm{n}=5)$, and ApoE KO-EX+TM $(\mathrm{n}=4) .(\mathbf{C}-\mathbf{H})$ : The protein expression of ER stress markers; GRP78 (C; $\mathrm{n}=6$ /groups), $\mathrm{p}$-IRE1 (D; $\mathrm{n}=6$ /groups), t-IRE1 (E; $\mathrm{n}=7$ /groups), p-eIF2 $\alpha$ (F; n=6/groups), t-eIF2 $\alpha$ (G; $n=8$ /groups), CHOP $(\mathbf{H} ; \mathrm{n}=5$ /groups), and pro-apoptotic marker, Bax (I; $\mathrm{n}=7$ /groups) were analyzed by western blot. Values are presented as means \pm SEM. ${ }^{*} p<0.05$ versus WT, ${ }^{*} p<0.05$ versus ApoE KO, ${ }^{s} p<0.05$ versus ApoE KO-EX. 


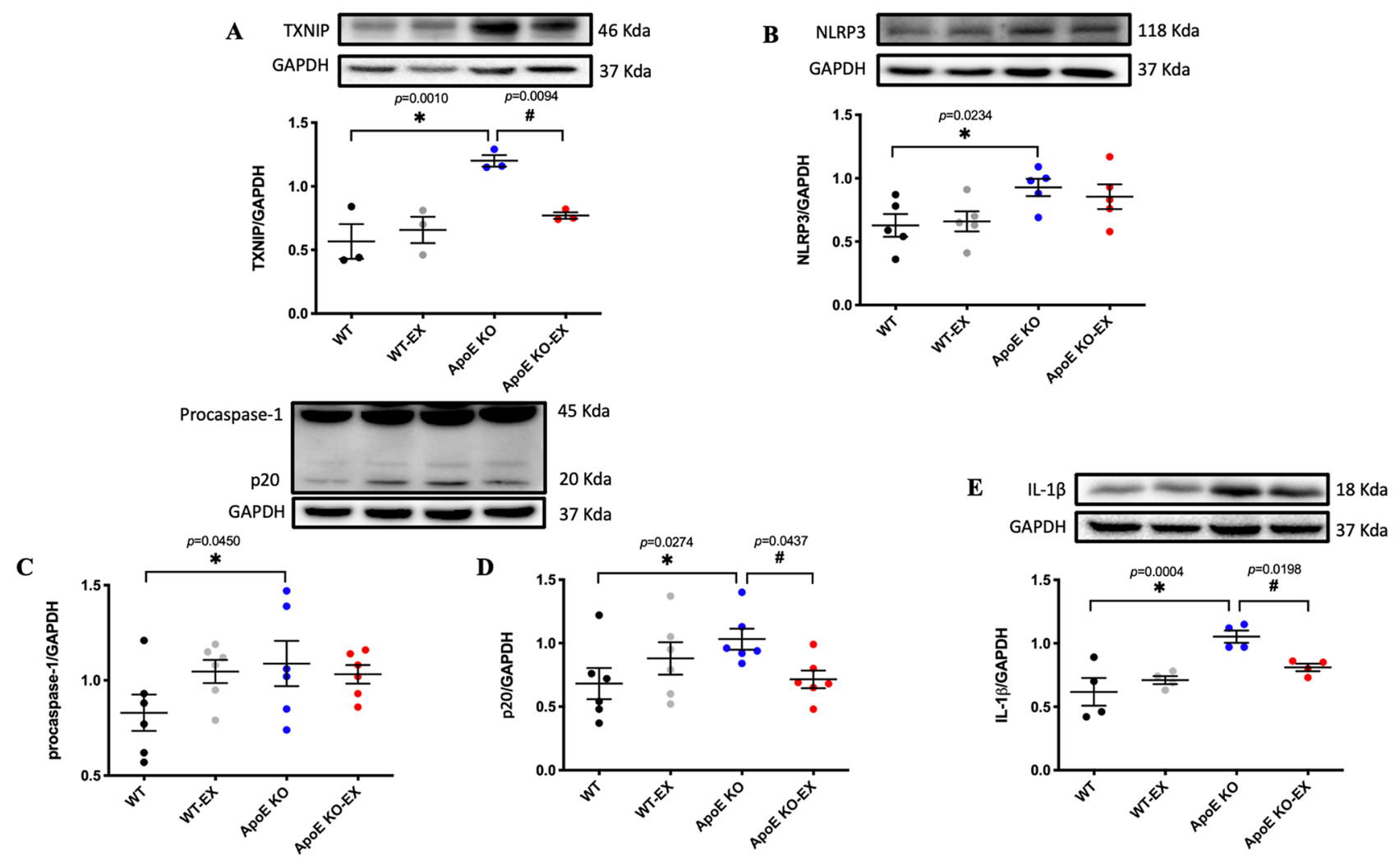

Figure 3. Effect of exercise training on TXNIP/NLRP3 inflammasome in the heart of ApoE KO mice. (A-E): The protein expression of TXNIP (A; $\mathrm{n}=3$ /groups), NLRP3 (B; $\mathrm{n}=5$ /groups), pro-caspase-1 (C; $\mathrm{n}=6$ /groups), caspase-1 p20 (D; n=6/groups), and IL-1 $\beta$ (E; n=4/groups) were measured by immunoblot analysis from each group. Bar graph values are presented as means \pm SEM. ${ }^{*} p<0.05$ versus $W T,{ }^{\#} p<0.05$ versus ApoE KO.

\section{Discussion}

Our results demonstrate that the cardiovascular benefits of exercise are intimately linked to the relationship between ER stress, UCP2, NLRP3 inflammasome, and oxidative stress. Specifically, our results indicate that exercise training improved endothelial function in coronary arterioles of ApoE KO mice through NO-dependent signaling pathways. We further showed that exercise training ameliorated ER stress-associated endothelial dysfunction in coronary arterioles in ApoE KO mice by reducing ER stress and TXNIP/NLRP3 inflammasome signaling. Lastly, we determined that exercise training rescued UCP2 deficiency-mediated endothelial dysfunction in coronary arterioles of ApoE KO via down-regulation of ROS by means of up-regulating UCP2 expressions.

Exercise training has emerged as a potentially significant therapeutic strategy for the prevention and treatment of atherosclerosis ${ }^{20}$. These positive changes are directly linked to the amelioration of endothelial dysfunction by the preservation of NO bioavailability ${ }^{21,22}$. We reported in the previous study that exercise training reduced atherosclerotic plaque formation in the aorta from ApoE KO mice model ${ }^{18}$. More importantly, the current study showed that exercise training improved the ACh-induced endothelial function in coronary arterioles of ApoE KO mice (Fig. 1A-E) through endothelium-dependent NO signaling pathways. These NO-mediated beneficial effects of exercise on vascular function in atherosclerosis have been well-matched with a number of the previous studies ${ }^{21-23}$. However, our nitrite/nitrate assay data has a limitation to determine NO bioavailability. The method is an indirect quantitative determination of NO concentration by measuring total nitrite and nitrate concentration.

ER stress is a crucial factor for modulating endothelial dysfunction by regulation of oxidative stress, inflammation, and NO bioavailability in atherosclerosis ${ }^{4}$. Exercise training has been shown to be an effective therapeutic intervention for reducing ER stress burden in a variety of disease models ${ }^{24}$. Recently, vascular functional studies have shown that ER stress is a critical player in endothelial dysfunction by reducing eNOS expression and NO bioavailability in ApoE KO mice and other pathological animal models ${ }^{17,18,25,26}$. Our vascular functional data confirmed that ER stress induction directly impaired endothelial function in coronary arterioles of WT and ApoE KO-EX mice, but no further impairment was shown in ApoE KO mice (Fig. 2A,B). Also, exercise training effectively suppressed the elevated ER stress markers (Fig. 2C-H). Furthermore, exercise training positively counteracted the expression of the CHOP-mediated pro-apoptosis marker, Bax (Fig. 2I). Our findings are validated by the literature which shows that regular exercise training ameliorates ER stress-mediated endothelial dysfunction through the down-regulation of ER stress markers in mesenteric arteries from diabetic ${ }^{17}$ and ApoE KO mice ${ }^{18}$. Recent studies reported that the absence of peroxisome proliferator-activated receptor- $\delta$ (PPAR- $\delta$ ) and PDE 5 abolished the exercise-induced beneficial changes to ER stress and NO-mediated vasodilatory signaling pathways in diabetes and aged animal models ${ }^{17,27}$. It suggested that exercise training reduces ER stress burden and increases 
A

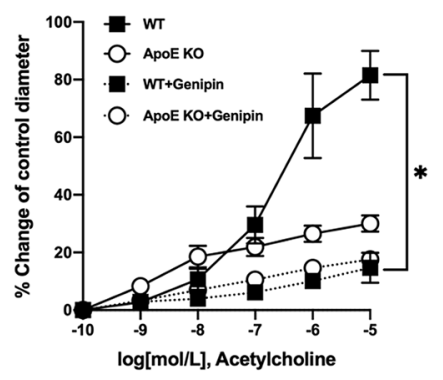

D

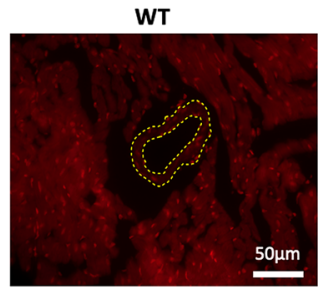

DHE

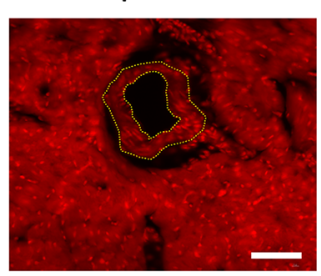

F

PECAM

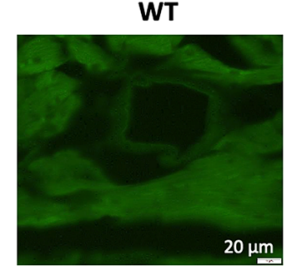

p67phox
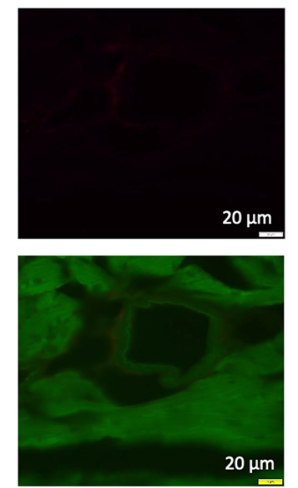

B

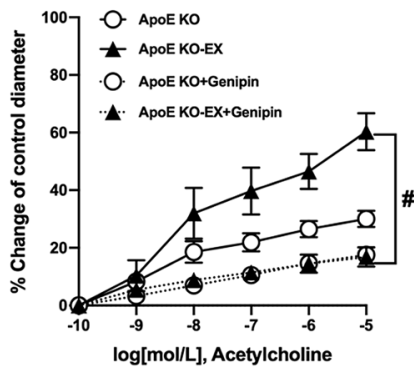

WT-EX

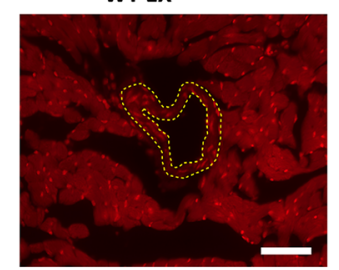

APOE KO+EX

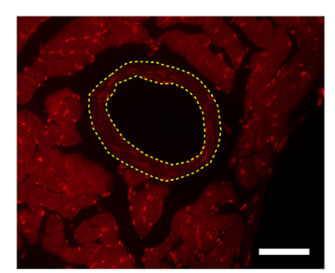

C
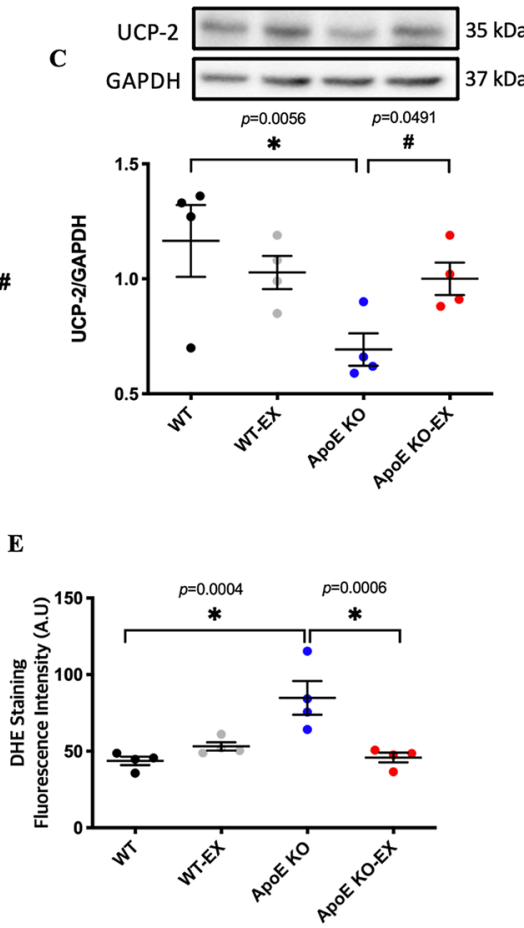

WT-EX
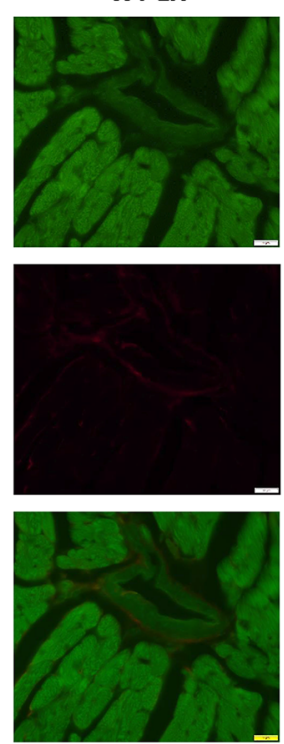
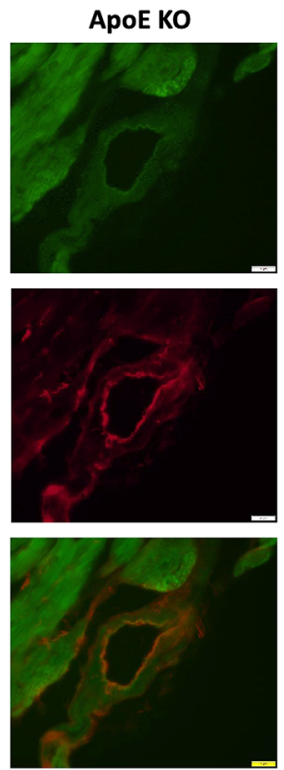

ApoE KO+EX
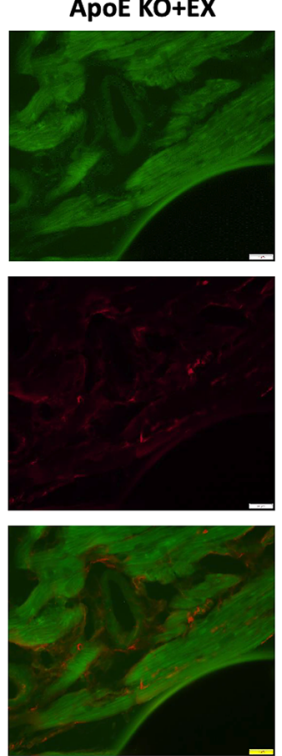

Figure 4. Effect of exercise training on UCP2 deficiency-mediated endothelial dysfunction in coronary arterioles and oxidative stress in the heart of ApoE KO mice. (A,B): ACh-induced vasodilation was measured in the present of UCP2 inhibitor (genipin) from isolated coronary arterioles in WT + genipin $(\mathrm{n}=4)$, WT-EX+genipin $(n=4)$, ApoE KO + genipin $(n=5)$ and ApoE KO-EX + genipin $(n=4)$. (C): The protein expression of UCP2 was measured by immunoblot analysis from each group ( $n=4 /$ groups). (D): Representative images of DHE staining from each group. Magnification: $\times 20$. Scale bar $=50 \mu \mathrm{m}$. (E): Quantified DHE intensity from four independent experiments using the different samples $(n=4$ /groups). (F): Immunofluorescence image of NADPH oxidase subunit p67phox in the coronary arterioles. Magnification: $\times 40$. Scale bar $=20 \mu \mathrm{m}$. Values are presented as means \pm SEM. ${ }^{*} p<0.05$ versus $W T,{ }^{\#} p<0.05$ versus ApoE KO. 
NO bioavailability via the regulation of PPAR- $\delta$ and $c G M P / P K G / P D E 5$ signaling. Collectively, we believe our current study may support a causal link between exercise training and improved NO-mediated endothelial function through the down-regulation of ER stress in ApoE KO mice (Fig. 2). This might occur through the improvement of PPAR- $\delta$ and cGMP/PKG/PDE5 signaling cascades, which in turn, activate the reduction of the ER stress, improve vasorelaxation by elevation of NO concentration, and induces cellular survival signaling. Furthermore, a treatment of ER stress inhibitor would demonstrate the solid causal link between ER stress and endothelial impairment in coronary arterioles in ApoE KO mice and it should be addressed in the future study.

The ER stress-induced inflammatory response in endothelial cells is mediated through the activation of the TXNIP/NLRP3 inflammasome signaling cascade ${ }^{28}$. The TXNIP/NLRP3 inflammasome signaling is highly associated with the initiation and progression of atherosclerosis ${ }^{29}$. Previous vascular functional studies confirmed that NLRP3 inflammasome plays a pivotal role in the regulation of endothelial dysfunction by activation of caspase-1, IL-1 $\beta$, and IL-18 in metabolic disease models ${ }^{18,30,31}$. Again, exercise is an established intervention for inflammation-induced vascular dysfunction by promoting anti-inflammatory milieu in cardiovascular disease $\mathrm{e}^{32}$, however, very little data exists regarding the effect of exercise on TXNIP/NLRP3 inflammasome signaling in atherosclerosis. Our findings show that exercise training reduces the expression of TXNIP and caspase-1 p20, IL-1 $\beta$ in ApoE KO (Fig. 3A-E). Also, exercise training reduces the elevated expression of TXNIP, NLRP3, and its downstream cascade in obese mice mode ${ }^{19}$ and in the $A \beta$-injected mice ${ }^{33}$. In further support, the blocking of NLRP3 restores eNOS expression and NO production by reducing expression of NLRP3, and thereby eliminating the downstream products of its activation in ApoE $\mathrm{KO}^{34}$ and diabetic mouse models ${ }^{35}$. Collectively, our current findings suggest that the elimination of TXNIP/NLRP3 inflammasome signaling by exercise might be a key mechanism for improving coronary endothelial function along with ER stress. However, further studies are necessary to investigate the causal relationship between exercise training and ER stress-induced TXNIP/NLRP3 inflammasome signaling in coronary arterioles in atherosclerosis.

UCP2 is one of the major antioxidant proteins dampening mitochondria-derived ROS production ${ }^{5}$, in turn, regulating NO bioavailability. The deficiency of UCP2 is associated with the initiation and progression of atherosclerosis $^{7}$ and endothelial dysfunction with increased ROS and reduced NO expression ${ }^{6,36}$. The beneficial effect of exercise on the regulation of UCP2 expression and oxidative stress is well described in peripheral blood mononuclear cells (PBMCs) and in the aorta of aged rats ${ }^{37}$. However, there is very little data regarding how exercise influences UCP2 signaling in atherosclerosis. Our vascular functional data show that the treatment of UCP2 inhibitor, genipin, revoked the exercise benefit they gained in ApoE KO-EX, and the reduced protein expression of UCP2 was increased by exercise training (Fig. 4). Our findings suggest that exercise training could attenuate UCP2 deficiency-mediated endothelial dysfunction possibly through the elevation of UCP2 expression. The elevation of UCP2 expression by exercise training occurs through the PGC1 $\alpha /$ PPAR- $\delta$ pathway, which regulates ROS generation and increases eNOS expression ${ }^{38-40}$. Also, the impaired endothelial function was attenuated by up-regulation of UCP2 along with the elevation of eNOS expression/NO bioavailability and the reduction of ROS in human aortic endothelial cells (HAECs) and rat aorta ${ }^{41}$. It suggested that the activation PGC1 $\alpha / P P A R-\delta$ pathway by exercise increases UCP2 expression, which in turn, reduces ROS generation and elevates NO bioavailability. Likewise, exercise reduced the elevated superoxide production and NADPH oxidase expression in the coronary arterioles of ApoE KO mice (Fig. 4D-F). Accordingly, our findings suggest that the upregulation of UCP2 expression by exercise training ameliorates vascular dysfunction through the reduction of UCP2 deficiency-derived ROS production in atherosclerosis and it might be regulated by PGC1 $\alpha / \mathrm{PPAR}-\delta$ signaling.

The current study can provide a more comprehensive understanding of the beneficial effect of exercise on cardiovascular diseases in atherosclerosis. We have reported the protective effect of exercise training on ER stress-mediated endothelial dysfunction in mesenteric arteries in atherosclerosis ${ }^{18}$. In addition, here we report that exercise training ameliorates ER stress and UCP2 deficiency-associated endothelial dysfunction in different vascular beds, coronary arterioles where mainly regulate blood flow to the cardiac muscle, in atherosclerosis. Also, we determine more in-depth underlying molecular mechanisms by which exercise training improves coronary endothelial function by regulating ER stress, TXNIP/NLRP3 inflammasome signaling cascades, and UCP2-regulated ROS generation in atherosclerosis. In conclusion, our study reinforces that exercise training is a profoundly effective therapy for the treatment and prevention on ER stress and UCP2 deficiency-associated coronary arteriole dysfunction in atherosclerosis via regulation of inflammation and oxidative stress including TXNIP/NLRP3 inflammasome and UCP2 deficiency-mediated ROS generation. However, further causal mechanistic studies are required to decisively establish how exercise influences these changes.

\section{Methods}

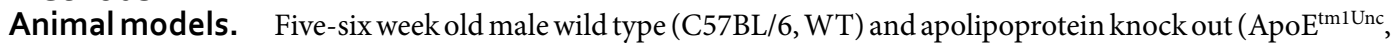
ApoE KO) mice were supplied from the Jackson Laboratory (Bar Harbor, ME). WT and ApoE KO mice were randomly divided into either exercise training (EX) or sedentary groups. Mice were housed in a temperaturecontrolled $\left(22-23^{\circ} \mathrm{C}\right)$ animal facility with $12 \mathrm{~h}$ of light/dark cycles and allowed free access to water and chow. WT and WT-EX mice were fed a normal chow and ApoE KO and ApoE KO-EX mice were fed a western atherogenic diet ( $0.2 \%$ cholesterol, $42 \% \mathrm{Kcal}$ from fat, TD.88137, ENVIGO) for 17 weeks to accelerate atherosclerosis development. All mice were sacrificed at 24-25 weeks old. All experimental protocols were approved by the Institutional Animal Care and Use Committee at the University of Houston (15-020). The described all experiments on animals were performed in agreement with the NIH Guidelines for the Use of Animals in Research and were carried out in compliance with the ARRIVE guidelines.

Exercise training protocols. The exercise training protocol was previously described in detail ${ }^{42}$. At 7-8 weeks of age, WT-EX and ApoE KO-EX mice were subjected to five consecutive days of aerobic exercise 
on a motorized rodent treadmill (Columbus Instruments, Columbus, OH). Preceding the exercise intervention, the EX mice were acclimated to running on a treadmill for 1 week. Each exercise training session consisted of $1 \mathrm{~h}$ of running on a motor-driven treadmill at $15 \mathrm{~m} / \mathrm{min}$ at a $5^{\circ}$ grade $\left(60 \sim 80 \%\right.$ of $\left.\mathrm{VO}_{2} \max \right), 5$ days/week for 15-16 weeks. The programmed exercise protocol consisted of warm-up (5 min), running (50 min), and cooldown ( $5 \mathrm{~min}$ ). Sedentary groups of mice remained in the same room and conditions throughout the experiment. At 24-25 weeks of age, all mice were sacrificed by $\mathrm{CO}_{2}$ inhalation and bilateral thoracotomy $24 \mathrm{~h}$ after the final exercise training session.

Functional assessment of isolated coronary arterioles of ApoE KO mice. After euthanasia, the heart was removed and rapidly placed in cold $\left(4^{\circ} \mathrm{C}\right)$ physiological saline solution (PSS). An isolated coronary arteriole $(60-100 \mu \mathrm{m}$ in internal diameter and $0.5-1.0 \mathrm{~mm}$ in length) was cannulated with glass micropipettes filled with PSS-albumin solution, and secured with surgical nylon sutures. The chamber was transferred to a stage of an inverted microscope (Nikon Eclipse Ti-S) and the cannulated arteriole was pressurized to $60 \mathrm{cmH}_{2} \mathrm{O}$ intraluminal pressure without the flow. After developing a stable basal tone $(1 \mathrm{~h})$, the vasodilation function was tested by different stimuli, as previously described ${ }^{43}$. The dose-dependent-diameter changes were established with an endothelium-dependent vasodilator, $\mathrm{ACh}(0.1 \mathrm{nmol} / \mathrm{L}$ to $10 \mu \mathrm{mol} / \mathrm{L})$ in coronary arterioles. Tunicamycin (ER stress inducer, TM, $10 \mu \mathrm{mol} / \mathrm{L}$ ), and genipin (UCP-2 inhibitor, $10 \mu \mathrm{mol} / \mathrm{L}$ ) were each incubated with the isolated vessel for 20-30 min before measuring ACh-induced endothelium-dependent vasodilation. The contribution of NO to vasodilation was assessed by incubating the vessels with the $N^{G}$-nitro-L-arginine methyl ester (L-NAME; NO synthases inhibitor; $10 \mu \mathrm{mol} / \mathrm{L}, 20 \mathrm{~min}$ ). TM was dissolved in $0.1 \% \mathrm{DMSO}$ and all other drugs were dissolved in PSS and then extraluminally administrated into the PSS in the chamber before measuring the vascular function. At the end of each experiment, the endothelium-independent vasodilator; sodium nitroprusside (SNP, $0.1 \mathrm{nmol} / \mathrm{L}$ to $10 \mu \mathrm{mol} / \mathrm{L}$ ) was applied to the vessel to obtain smooth muscle-dependent vasodilation and then the vessel was exposed to $100 \mu \mathrm{mol} / \mathrm{L} \mathrm{SNP}$ to obtain its maximal diameter at $60 \mathrm{cmH}_{2} \mathrm{O}$ intraluminal pressure. These assessments were accomplished in coronary arterioles from animals assigned to all four experimental groups.

Measurement of intracellular superoxide $\left(\mathrm{O}_{2}{ }^{-}\right)$. To evaluate the production of superoxide in coronary arterioles, the oxidative fluorescent dye dihydroethidium (DHE) was used. Frozen cardiac tissue embedded in OCT compound was sectioned at $10 \mu \mathrm{m}$ thickness at $-25^{\circ} \mathrm{C}$ using a cryostat (Leica CM 1950, Leica Biosystems Inc., Buffalo Grove, IL). The sections were placed on microscope slides, and the slides were dried at RT for 15 min. The slides in PBS were then incubated in a light-protected humidified incubator at $37{ }^{\circ} \mathrm{C}$ with $95 \% \mathrm{O}_{2}$ and $5 \% \mathrm{CO}_{2}$ for $1 \mathrm{~h}$, after which the slides were incubated with $5 \mu \mathrm{m}$ DHE for $15 \mathrm{~min}$ at RT in a dark room. Finally, the slides were washed 3 times in cold PBS (every $5 \mathrm{~min}$ ) and were mounted with a cover slide in the darkroom. DHE stained images were visualized by an Olympus BX51 fluorescence microscope and acquired with 20X objective lens at an excitation peak of $545 \mathrm{~nm}$ with an emission spectral peak of $610 \mathrm{~nm}$. Fluorescence intensity was measured by Image J.

Measurement of total nitrate/nitrite concentration in heart tissue. Myocardial nitrate/nitrite concentration was estimated using a Colorimetric Assay Kit (78001, Cayman Chemical, Ahn Arbor, MI), as per the manufacturer's instruction. To determine the total nitrate/nitrite level in the supernatant solution, the samples were duplicated and measured by a colorimetric microplate reader at $540 \mathrm{~nm}$.

Immunoblotting. Heart tissue was homogenized and lysed in RIPA buffer with protease/phosphatase inhibitor cocktails, and then centrifuged $15,000 \mathrm{~g}$ for $20 \mathrm{~min}$ at $4{ }^{\circ} \mathrm{C}$ and protein concentration was measured by BCA assay. Proteins from heart and cell lysates ( $30 \mu \mathrm{g}$ per sample) were resolved by Tris-glycine sodium dodecyl sulphate-polyacrylamide (SDS-PAGE) gel electrophoresis and transferred to polyvinylidene difluoride (PVDF) membrane. Subsequently, the membrane was blocked with $5 \%$ non-fat milk in Tris-buffered saline-Tween 20 $(0.05 \% \mathrm{TBST})$ for $30 \mathrm{~min}$ at RT and incubated overnight with respective primary antibodies. The primary antibodies were purchased from the following sources: TXNIP (Santa Cruz; sc-271237), NLRP3 (Abcam; ab4207), caspase-1 (Abcam; ab108362), IL-1 $\beta$ (Abcam; ab9722), p-IRE1 (Abcam; ab48187), IRE1 (Abcam; ab37073), p-eIF2 $\alpha$ (Invitrogen; 44728G), eIF2 $\alpha$ (Cell Signaling; 9722), CHOP (Invitrogen; MA1-250), Bax (Cell Signaling; 2772), UCP-2 (Santa Cruz; sc-390189), and GAPDH (Cell Signaling; sc47778). The membrane was then washed three times in TBST and incubated with horseradish peroxidase (HRP)-conjugated secondary antibodies for two hours at RT followed by washing three times with TBST. The blot bands were developed with enhanced chemiluminescence (Thermo Fisher Scientific Inc., MA, USA) for visualization, detected by ChemiDoc ${ }^{\mathrm{Ts}} \mathrm{MP}$ imager (Bio-Rad, CA), and quantified using NIH ImageJ software for densitometric analysis. Protein expression was normalized to the internal control, GAPDH. The full-length of the membrane was not able to provide because the blot was cut prior to incubating primary antibodies. All blots with membrane edges are provided in the Supplementary file.

Immunofluorescence. Immunofluorescence experiments were performed as previously described with some modifications ${ }^{19,44}$ in order to determine $\mathrm{p} 67^{\text {phox }}$ localization on the endothelium of coronary arterioles with diameters ranging from 50 to $100 \mu \mathrm{m}$. In brief, the freshly frozen heart was sectioned at $10 \mu \mathrm{m}$. After $1 \mathrm{~h}$ of acetone permeabilization, slides were incubated with a $10 \%$ goat serum in PBS for 30 min at RT after three washes in PBS. A mouse on mouse (Vector Laboratories) kit was used to prevent non-specific binding between testing tissue and an antibody from mouse host. The sections were then incubated for one hour at RT in a blocking buffer with primary antibodies, including PECAM1 (Abcam) and anti-p67 $7^{\text {phox }}$ (BD Biosciences). After 
three washes in PBS, sections were incubated with the fluorescence-conjugated secondary antibody, Alexa Fluor 488 and Alexa Fluor 594 (Thermo Fischer Scientific) and mounted with ProloLong antifade solution. Negative controls were performed with the use of mouse $\operatorname{IgG}_{2 b}$ and rabbit IgG (Santa Cruz Biotechnology). Images were taken with an Olympus BX41 fluorescence microscope, using $40 \times$ objective. Microscope and camera settings were consistent throughout the process for continuity of data for all groups of samples.

Data analysis. All data are presented as mean \pm SEM. All diameter changes to pharmacological agonists were normalized to the control diameter. Statistical comparisons of vasoreactivity responses between groups were performed with two-way analysis of variance (ANOVA) for repeated measure and intergroup differences were tested with Fisher's protected LSD test. The significance of intergroup differences observed in body weight, vessel diameters, and protein expressions were analyzed with one-way analysis of variance (one-way ANOVA) using software SPSS 22.0. Significance was accepted at $p<0.05$.

\section{Data availability}

The data that supports the findings of this study are available from the corresponding author upon reasonable request.

Received: 11 March 2021; Accepted: 8 July 2021

Published online: 29 July 2021

\section{References}

1. Garcia, M. C. et al. Potentially excess deaths from the five leading causes of death in metropolitan and nonmetropolitan counties-United States, 2010-2017. MMWR Surveill. Summ. 68, 1-11. https://doi.org/10.15585/mmwr.ss6810a1 (2019).

2. Chen, J. Y. et al. Nitric oxide bioavailability dysfunction involves in atherosclerosis. Biomed. Pharmacother. 97, 423-428. https:// doi.org/10.1016/j.biopha.2017.10.122 (2018).

3. Theodorou, K. \& Boon, R. A. Endothelial cell metabolism in atherosclerosis. Front. Cell Dev. Biol. 6, 82. https://doi.org/10.3389/ fcell.2018.00082 (2018).

4. Lenna, S., Han, R. \& Trojanowska, M. Endoplasmic reticulum stress and endothelial dysfunction. IUBMB Life 66, 530-537. https:// doi.org/10.1002/iub.1292 (2014).

5. Droge, W. Free radicals in the physiological control of cell function. Physiol. Rev. 82, 47-95. https://doi.org/10.1152/physrev.00018. $2001(2002)$

6. Tian, X. Y., Ma, S., Tse, G., Wong, W. T. \& Huang, Y. Uncoupling protein 2 in cardiovascular health and disease. Front. Physiol. 9, 1060. https://doi.org/10.3389/fphys.2018.01060(2018).

7. Blanc, J. et al. Protective role of uncoupling protein 2 in atherosclerosis. Circulation 107, 388-390 (2003).

8. Sreedhar, A. \& Zhao, Y. Uncoupling protein 2 and metabolic diseases. Mitochondrion 34, 135-140. https://doi.org/10.1016/j.mito. 2017.03.005 (2017).

9. Zhou, R., Tardivel, A., Thorens, B., Choi, I. \& Tschopp, J. Thioredoxin-interacting protein links oxidative stress to inflammasome activation. Nat. Immunol. 11, 136-140. https://doi.org/10.1038/ni.1831 (2010).

10. World CJ, Yamawaki, H. \& Berk, B. C. Thioredoxin in the cardiovascular system. J. Mol. Med. (Berl) 84, 997-1003. https://doi.org/ 10.1007/s00109-006-0109-6 (2006).

11. Ogura, Y., Sutterwala, F. S. \& Flavell, R. A. The inflammasome: First line of the immune response to cell stress. Cell 126, 659-662. https://doi.org/10.1016/j.cell.2006.08.002 (2006).

12. Abderrazak, A. et al. NLRP3 inflammasome: from a danger signal sensor to a regulatory node of oxidative stress and inflammatory diseases. Redox Biol. 4, 296-307. https://doi.org/10.1016/j.redox.2015.01.008 (2015)

13. Yin, Y. et al. Vascular endothelial cells senescence is associated with NOD-like receptor family pyrin domain-containing 3 (NLRP3) inflammasome activation via reactive oxygen species (ROS)/thioredoxin-interacting protein (TXNIP) pathway. Int. J. Biochem. Cell Biol. 84, 22-34. https://doi.org/10.1016/j.biocel.2017.01.001 (2017).

14. Zhang, Y. et al. Coronary endothelial dysfunction induced by nucleotide oligomerization domain-like receptor protein with pyrin domain containing 3 inflammasome activation during hypercholesterolemia: Beyond inflammation. Antioxid. Redox Signal. 22, 1084-1096. https://doi.org/10.1089/ars.2014.5978 (2015).

15. Miteva, K., Madonna, R., De Caterina, R. \& Van Linthout, S. Innate and adaptive immunity in atherosclerosis. Vascul. Pharmacol. https://doi.org/10.1016/j.vph.2018.04.006 (2018).

16. Pereira, C. A., Carneiro, F. S., Matsumoto, T. \& Tostes, R. C. Bonus effects of antidiabetic drugs: Possible beneficial effects on endothelial dysfunction, vascular inflammation and atherosclerosis. Basic Clin. Pharmacol. Toxicol. 123, 523-538. https://doi.org/ 10.1111/bcpt.13054 (2018).

17. Cheang, W. S. et al. PPARdelta is required for exercise to attenuate endoplasmic reticulum stress and endothelial dysfunction in diabetic mice. Diabetes 66, 519-528. https://doi.org/10.2337/db15-1657 (2017).

18. Hong, J. et al. Exercise ameliorates endoplasmic reticulum stress-mediated vascular dysfunction in mesenteric arteries in atherosclerosis. Sci. Rep. 8, 7938. https://doi.org/10.1038/s41598-018-26188-9 (2018).

19. Lee, J. et al. Physical activity protects NLRP3 inflammasome-associated coronary vascular dysfunction in obese mice. Physiol. Rep. 6, e13738. https://doi.org/10.14814/phy2.13738 (2018).

20. Okabe, T. A. et al. Swimming reduces the severity of atherosclerosis in apolipoprotein E deficient mice by antioxidant effects. Cardiovasc. Res. 74, 537-545. https://doi.org/10.1016/j.cardiores.2007.02.019 (2007).

21. Guizoni, D. M. et al. Aerobic exercise training protects against endothelial dysfunction by increasing nitric oxide and hydrogen peroxide production in LDL receptor-deficient mice. J. Transl. Med. 14, 213. https://doi.org/10.1186/s12967-016-0972-z (2016).

22. Pellegrin, M. et al. New insights into the vascular mechanisms underlying the beneficial effect of swimming training on the endothelial vasodilator function in apolipoprotein E-deficient mice. Atherosclerosis 190, 35-42. https://doi.org/10.1016/j.ather osclerosis.2006.02.001 (2007).

23. Terra, M. F., Pedrosa, D. G., Zoppi, C. C., Werneck, C. C. \& Vicente, C. P. Physical exercises decreases thrombus and neointima formation in atherosclerotic mice. Thromb. Res. 175, 21-31. https://doi.org/10.1016/j.thromres.2019.01.003 (2019).

24. Hong, J., Kim, K., Kim, J. H. \& Park, Y. The role of endoplasmic reticulum stress in cardiovascular disease and exercise. Int. J. Vasc. Med. 2017, 2049217. https://doi.org/10.1155/2017/2049217 (2017).

25. Lau, Y. S. et al. 3',4'-dihydroxyflavonol ameliorates endoplasmic reticulum stress-induced apoptosis and endothelial dysfunction in mice. Sci. Rep. 8, 1818. https://doi.org/10.1038/s41598-018-19584-8 (2018).

26. Kassan, M. et al. Endoplasmic reticulum stress is involved in cardiac damage and vascular endothelial dysfunction in hypertensive mice. Arterioscler. Thromb. Vasc. Biol. 32, 1652-1661. https://doi.org/10.1161/ATVBAHA.112.249318 (2012). 
27. Chang, P. et al. Swimming exercise inhibits myocardial ER stress in the hearts of aged mice by enhancing cGMPPKG signaling. Mol. Med. Rep. 21, 549-556. https://doi.org/10.3892/mmr.2019.10864 (2020).

28. Mohamed, I. N. et al. Thioredoxin-interacting protein is required for endothelial NLRP3 inflammasome activation and cell death in a rat model of high-fat diet. Diabetologia 57, 413-423. https://doi.org/10.1007/s00125-013-3101-z (2014).

29. Hoseini, Z. et al. NLRP3 inflammasome: Its regulation and involvement in atherosclerosis. J. Cell Physiol. 233, 2116-2132. https:// doi.org/10.1002/jcp.25930 (2018).

30. Pereira, C. A. et al. Mitochondrial DNA promotes NLRP3 inflammasome activation and contributes to endothelial dysfunction and inflammation in type 1 diabetes. Front. Physiol. 10, 1557. https://doi.org/10.3389/fphys.2019.01557 (2019).

31. Ferreira, N. S. et al. NLRP3 inflammasome and mineralocorticoid receptors are associated with vascular dysfunction in type 2 diabetes mellitus. Cells 8, 4. https://doi.org/10.3390/cells8121595 (2019).

32. Fiuza-Luces, C. et al. Exercise benefits in cardiovascular disease: Beyond attenuation of traditional risk factors. Nat. Rev. Cardiol. 15, 731-743. https://doi.org/10.1038/s41569-018-0065-1 (2018).

33. Rosa, J. M., Camargo, A., Wolin, I. A. V., Kaster, M. P. \& Rodrigues, A. L. S. Physical exercise prevents amyloid beta1-40-induced disturbances in NLRP3 inflammasome pathway in the hippocampus of mice. Metab. Brain Dis. 36, 351-359. https://doi.org/10. 1007/s11011-020-00646-8 (2021).

34. Zheng, F., Xing, S., Gong, Z., Mu, W. \& Xing, Q. Silence of NLRP3 suppresses atherosclerosis and stabilizes plaques in apolipoprotein E-deficient mice. Mediators Inflamm. 2014, 507208. https://doi.org/10.1155/2014/507208 (2014).

35. Xing, J. H. et al. NLRP3 inflammasome mediate palmitate-induced endothelial dysfunction. Life Sci. 239, 116882. https://doi.org/ 10.1016/j.lfs.2019.116882 (2019).

36. Tian, X. Y. et al. Uncoupling protein-2 protects endothelial function in diet-induced obese mice. Circ Res 110, 1211-1216. https:// doi.org/10.1161/CIRCRESAHA.111.262170 (2012).

37. $\mathrm{Gu}, \mathrm{Q}$. et al. Chronic aerobic exercise training attenuates aortic stiffening and endothelial dysfunction through preserving aortic mitochondrial function in aged rats. Exp. Gerontol. 56, 37-44. https://doi.org/10.1016/j.exger.2014.02.014 (2014).

38. de Oliveira Bristot, V. J., de Bem Alves, A. C., Cardoso, L. R., da Luz Scheffer, D. \& Aguiar, A. S. Jr. The role of PGC-1alpha/UCP2 signaling in the beneficial effects of physical exercise on the brain. Front. Neurosci. 13, 292. https://doi.org/10.3389/fnins.2019. 00292 (2019).

39. Zhou, Y. et al. PPARgamma inhibits VSMC proliferation and migration via attenuating oxidative stress through upregulating UCP2. PLoS ONE 11, e0154720. https://doi.org/10.1371/journal.pone.0154720 (2016).

40. Wang, P. et al. Activation of PPAR-gamma by pioglitazone attenuates oxidative stress in aging rat cerebral arteries through upregulating UCP2. J. Cardiovasc. Pharmacol. 64, 497-506. https://doi.org/10.1097/FJC.0000000000000143 (2014).

41. Lee, K. U. et al. Effects of recombinant adenovirus-mediated uncoupling protein 2 overexpression on endothelial function and apoptosis. Circ. Res. 96, 1200-1207. https://doi.org/10.1161/01.RES.0000170075.73039.5b (2005).

42. Hong, J. et al. Exercise training ameliorates cerebrovascular dysfunction in a murine model of Alzheimer's disease: role of the P2Y2 receptor and endoplasmic reticulum stress. Am. J. Physiol. Heart Circul. Physiol. 318, H1559-H1569. https://doi.org/10.1152/ajphe art.00129.2020 (2020).

43. Park, Y., Booth, F. W., Lee, S., Laye, M. J. \& Zhang, C. Physical activity opposes coronary vascular dysfunction induced during high fat feeding in mice. J. Physiol. 590, 4255-4268. https://doi.org/10.1113/jphysiol.2012.234856 (2012).

44. Chakraborty, S. et al. Lipopolysaccharide modulates neutrophil recruitment and macrophage polarization on lymphatic vessels and impairs lymphatic function in rat mesentery. Am. J. Physiol. Heart Circul. Physiol. 309, H2042-H2057. https://doi.org/10. 1152/ajpheart.00467.2015 (2015).

\section{Author contributions}

J.H. and Y.P. conceived and designed the study. J.H., E.P., J.L., Y.L., and Y.P. conducted the experiments. J.H. and Y.P. analyzed the data. J.H. and Y.P. wrote the manuscript. J.H., E.P., J.L., Y.L., B.R., and Y.P. contributed to study design, data interpretation, and manuscript revision. All authors read and approved the final manuscript.

\section{Funding}

This study was supported by grants from the CardioVascular Research Foundation Asia (\#16215) and the College of Liberal Arts and Social Science of the University of Houston (Research Progress Grant) to Dr. Yoonjung Park.

\section{Competing Interests}

The authors declare no competing interests.

Additional information

Supplementary Information The online version contains supplementary material available at https://doi.org/ 10.1038/s41598-021-94944-5.

Correspondence and requests for materials should be addressed to Y.P.

Reprints and permissions information is available at www.nature.com/reprints.

Publisher's note Springer Nature remains neutral with regard to jurisdictional claims in published maps and institutional affiliations.

Open Access This article is licensed under a Creative Commons Attribution 4.0 International License, which permits use, sharing, adaptation, distribution and reproduction in any medium or format, as long as you give appropriate credit to the original author(s) and the source, provide a link to the Creative Commons licence, and indicate if changes were made. The images or other third party material in this article are included in the article's Creative Commons licence, unless indicated otherwise in a credit line to the material. If material is not included in the article's Creative Commons licence and your intended use is not permitted by statutory regulation or exceeds the permitted use, you will need to obtain permission directly from the copyright holder. To view a copy of this licence, visit http://creativecommons.org/licenses/by/4.0/.

(c) The Author(s) 2021 